\title{
Goal-Driven Workflow Generation Based on AI Planning
}

\author{
Yinxue Shi, Minghao Yang, and Ruizhi Sun \\ College of Information and Electrical Engineering, China Agricultural University, Beijing, \\ P.R. China \\ syx2821@gmail.com
}

\begin{abstract}
The success of an enterprise heavily depends on its ability to respond to changes in its environment in an effective and dynamic way. To cope with emerging business trends, automatic workflow generation provides a significant competitive advantage .This paper presents a goal-driven workflow generation, which is pattern-oriented and knowledge-based. First, a process pattern is proposed as a vehicle for knowledge representation. Then, a heuristic intelligent planning algorithm is deployed to construct a workflow automatically according to the goal specified by the users and the business context.
\end{abstract}

Keywords: goal-driven, pattern oriented, HTN planning, workflow generation.

\section{Introduction}

Process-driven workflow systems are used to configure and control structured business processes from which well-defined workflow models and instances can be derived [3,9]. Because all information needed in the execution stage is specified, this method is also called full-ahead plan [11]. However, since most business environments are complicated and changeable, it is difficult for users to know all the details for defining the process specification. And the process definitions often need to be modified during execution for the unexpected and developmental change in the business processes being modeled and the deviations from the prescribed process model at runtime [8].

Obviously, the traditional "full-ahead" $[12,13]$ workflow scheduling can't meet the complex and flexible enterprise. Automating the workflow generation process as much as possible is the main point. Ideally, the user just need to specify what they want the workflow to achieve (instead of how to achieve it) and what the constraint is. The workflow management system should then be able to generate an appropriate workflow and execute it successfully.

Presently, some research has focused on the workflow system that integrates AI planning techniques for automatic workflow construction or workflow generation. This method applying AI planning to dynamic workflow creation sacrifices the advanced features: Firstly, high availability is possible by enabling users to represent the workflows at an abstract level without needing to worry about the particulars of the target execution systems. Secondly, robustness is possible by generating a replaceable process when exception occurs. Because when the failure happens, real-time planning will find a new resolution to deal with the exception. But high scalability is required 
for the present planning algorithms usually limit to a specific application domain. Pegasus $[3,10]$ is a typical example which uses AI planning to workflow system in Grid environment.

Our work is different from the current practices in two aspects: the first is that a new workflow modeling method based on a pattern is proposed to specify a goaldriven workflow correctly. The second is that we adopt the heuristic evaluation algorithm and pattern-oriented HTN planning algorithm to knowledge representation and workflow planning, which enables the use of domain heuristics in problem solving and automatic workflow composition thus speeding up the planning process and improving the planner's performance. And it will lead a more effective way of exception handling and workflow simulation.

\section{Goal-Driven Workflow}

\subsection{Process-Driven Workflow}

Process-Driven Workflow largely takes the decision-making responsibility for the flow of documents, information and tasks out of the hands of the user, leaving it to an automated business process rules engine [4].

Process-Driven Workflow is a pre-defined workflow. Where the user should

* Understand and control the processes in detail.

* Identify the metrics and objectives for the key processes.

* Model and automate key business processes.

* Identify the participants who are involved in the process.

* Innovate continuously to keep pace with business change.

Usually, process-driven workflow functions well in a small-scale, centralized, homogeneous environment.

\subsection{Goal-Driven Workflow}

Goal-Driven Workflow is a more flexible workflow, where the boundary between workflow build-time and run-time is eliminated. Instead of defining a workflow process in advance, users just specify a goal and then workflow management system (WFMS) will, with or without users' intervention, decompose it into some sub-tasks according to the resources and knowledge possessed and fulfill the coordination and scheduling function necessary to achieve the specified goal. The decomposition procedure can be executed several times on the fly, making it possible to tolerate and/or adapt to the changes and exceptions encountered during the execution time [6].

Goal-Driven is more applicable in large-scale, distributed and heterogeneous environment. The users don't need to know the detail of process. They just need to describe what to be achieved.

\subsection{Process-Driven Workflow vs. Goal-Driven Workflow}

Table1 shows the differences between Process-Driven workflow and Goal-Driven workflow. Goal-Driven workflow has more advantages in real applications. 
Table 1. Process-Driven Workflow vs. Goal-Driven Workflow

\begin{tabular}{lll}
\hline & Process-Driven Workflow & Goal-Driven workflow \\
\hline style & full-ahead workflow & automatic construction, dynamic \\
application & special application domain & domain independent \\
definition & user based(understand the detail) & knowledge based \\
robustness & low & high \\
cost & low cost, multiple invest & single invest, knowledge maintenance \\
\hline
\end{tabular}

\section{Goal-Driven Workflow Generation}

\subsection{Process Pattern}

Patterns originally come from architectural domain to describe a problem which occurs over and over again in our environment, and then describes the workflow solutions to that problem, in such a way that you can use this solution a million times over [1].

Similarly, Process Pattern refers to the experiences and knowledge within business domain. It comprises three parts----Goal, Context and Solution which described as a triad:

$$
\text { Process Pattern }=<\text { Goal, Context, Solution }>
$$

Goal describes the target of business, whose properties include id, name and domain. Context describes the condition, whose properties include relevant data, positive factors and negative factors. Solution gives the guideline or solution to reach the

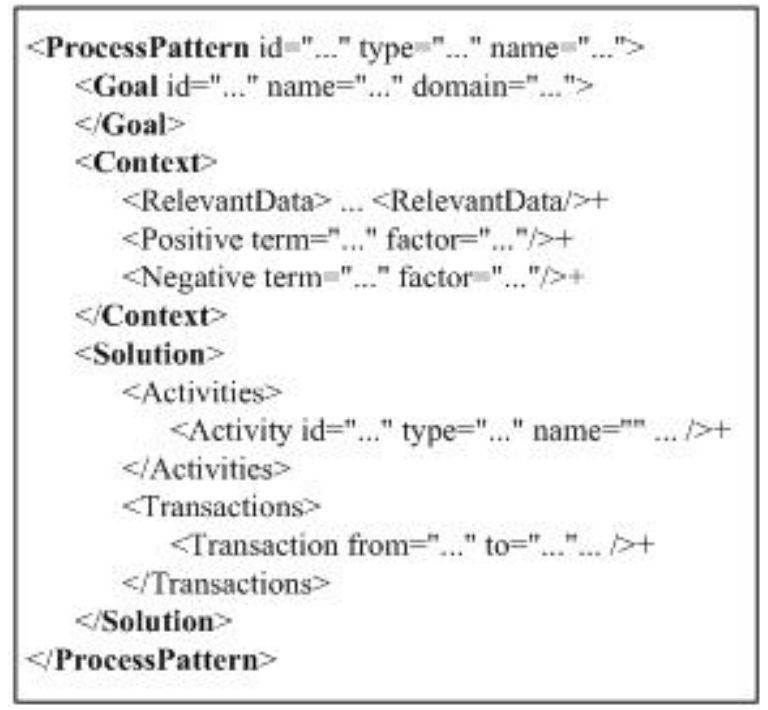

Fig. 1. Process Pattern in XML 
business goal. For the same goal, different context or scenario leads to different solutions. And each goal can be decomposed into several sub-goals. In the same way, sub-goal has its appropriate process pattern.

\subsection{Goal-Driven Workflow Generation}

Workflow generation means the automatic construction of workflow definition. In more detail, it includes the construction of definition, the refinement and the replanning in execution. Goal-driven workflow generation is based on process pattern. It can construct a workflow automatically according to the goal specified by the users and the business context.

The procedure of Goal-Driven workflow generation is shown as Fig. 2:

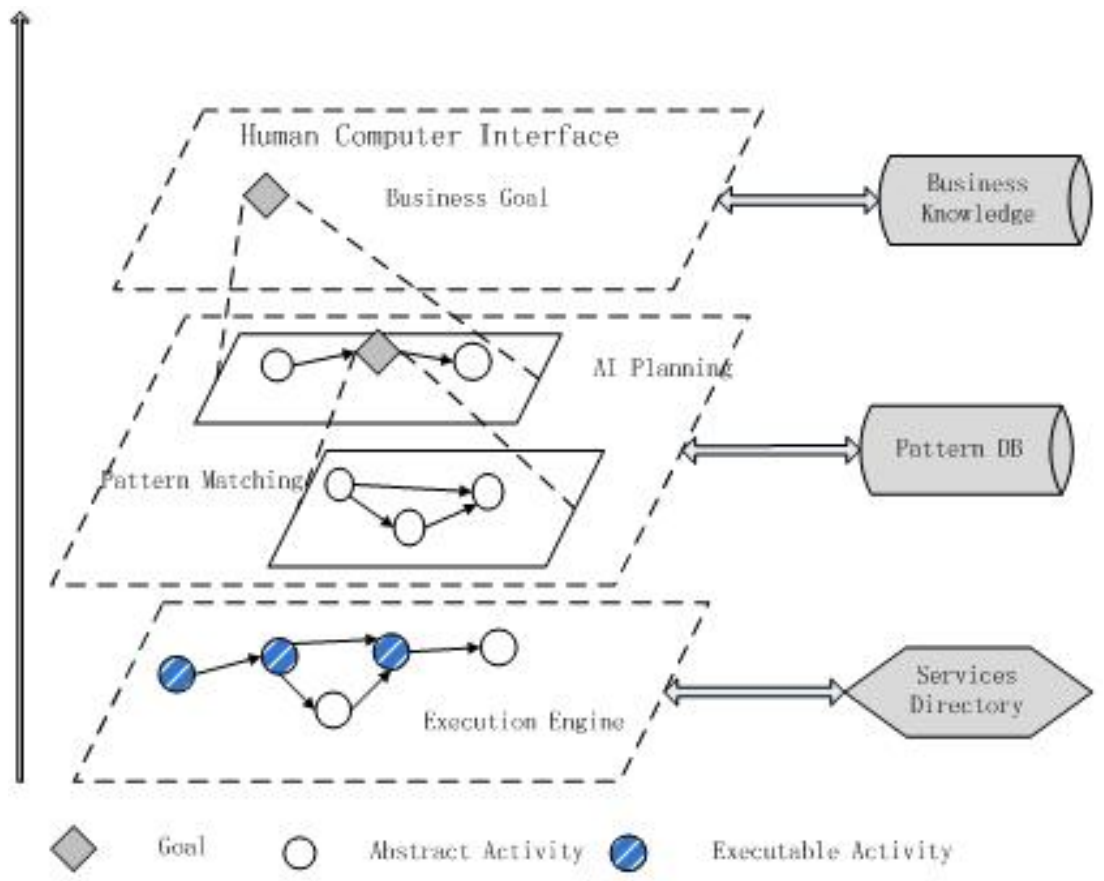

Fig. 2. Goal-Driven workflow generation

When modeling, the business process will be divided into a serious of process segments which can be represented with process pattern. All of these patterns are saved as knowledge. Once the user input the goal, workflow generation process starts. Then AI planner constructs a planning tree composed with patterns which are selected through goal matching and pattern matching. Continually, all of these tree nodes are arranged into a whole process definition according to the connection between patterns. Finally, the Execution Engine maps the identified process steps to the physical services and coordinates their execution. 


\section{AI Planning}

\subsection{HTN Planning}

In HTN planning a planning problem is represented by sets of tasks (task networks). Methods decompose non-primitive tasks into sub-tasks until a level of primitive tasks is reached, which can be solved by operators [7]. HTN planning decomposes highlevel tasks into simpler ones until eventually all tasks have been decomposed into actions. This feature makes HTN planning a promising means to realize Goal-Driven workflow generation. Because the Goal-driven workflow generation is based on process pattern, which can describe knowledge at different levels of abstraction resolved to various levels of detail.

As illustrated in the last section, Goal-Driven workflow generation allow userdefined process segments which named Process Pattern, and then use AI planner automatically to search appropriate patterns and auto-assembly to form the segments to a complete solution for the goal. A Heuristic intelligent planning algorithm is deployed to complete automatic workflow generation.

\subsection{Heuristic Intelligent Planning Algorithm}

Once user input the Goal and Context information from the human computer interface, heuristic intelligent planning module starts. The system finds the best solutions through goal matching, context matching and pattern matching. And then automatically assemble the process fragments into a complete workflow process. Fig. 3 shows the flow chart of the intelligent planning algorithm.

As shown in Fig. 3, HTN planning algorithm is described as follows:

Step 1: Input a planning problem $\mathrm{G}$

Step 2: If $\mathrm{G}$ contains only primitive tasks, then return the result

Step 3: Choose a goal task $\mathrm{g}$ in $\mathrm{G}$ in depth-first search traversal method

Step 4: Choose an expansion for $\mathrm{g}$, using the heuristic evaluation algorithm

Step 5: Replace g with the expansion

Go to step 2

HTN planning decomposes tasks until a plan consists only of primitive tasks. Expanding each non-primitive task (step 3-5) is done by choosing an appropriate reduction, which specifies one possible way of accomplishing that task. Reductions are stored as solutions or process, which associate non-primitive tasks or goals with task networks. In step 3, we have adopted a depth-first traversal method to expand the nodes to form a final complete solution. The algorithm is sound because a heuristic evaluation algorithm is used in the step 4.

The heuristic evaluation algorithm is an artificial neural network algorithm optimized by genetic algorithm.

Artificial Neural Networks (ANNs) are computational tools that have found extensive utilization in solving many complex real-world problems. The attractiveness of ANNs comes from their remarkable information processing characteristics pertinent mainly to nonlinearity, high parallelism, fault and noise tolerance, and learning and generalization capabilities [5]. 


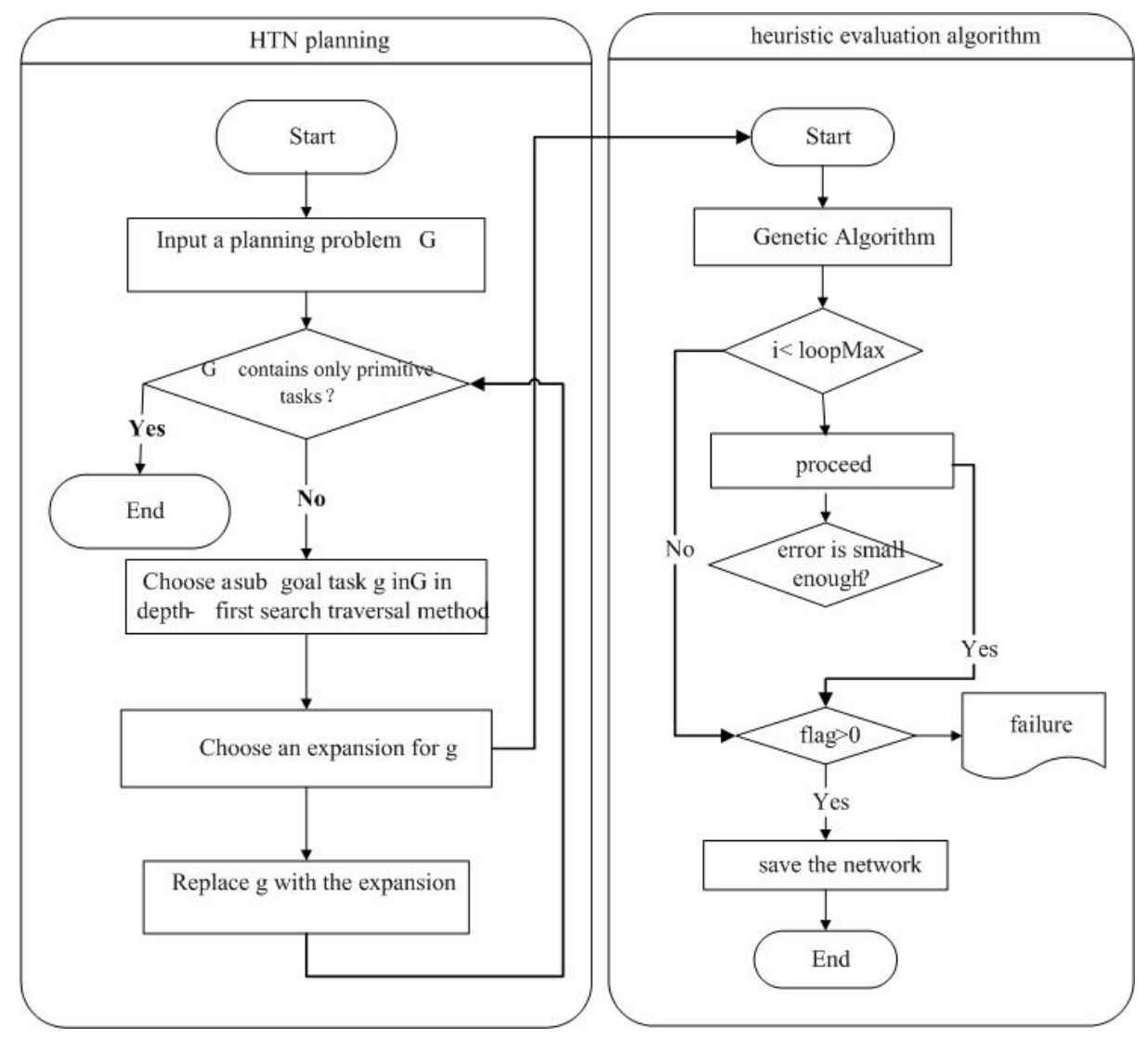

Fig. 3. Heuristic Intelligent Planning Algorithm

Artificial Neural Network algorithm is a kind of human brain simulation of the physical structure. That is to say, it uses computer methods to simulate the human brain from physical structure to make the system intelligent. Among the ANN models, multi-layer feed-forward neural network model is the most widely used model and often be trained by use of back-propagation learning algorithm (BP algorithm for short) . BP algorithm has the advantage of simple and plastic, but the BP algorithm is gradient-based method, which slow the convergence rate, and often affected by the local minima problem. Genetic Algorithm can get out of this predicament.

The following is the description of the heuristic evaluation algorithm:

Step 1: Use Genetic Algorithm to train Artificial Neural Network weights with reasonable cases set up by experts and spread the signal

Step 2: end the training if errors meet the condition and jump to Step 6

Step 3: the training error back-propagation

Step 4: modify network weights based on the results of the error back-propagation and jump to Step 2

Step 5: save the network weight and put it into use 
In step1, the Genetic Algorithm was used to train ANN weights. The procedure includes:

Step 1: randomly generate initial population

Step 2: access and sort the initial population

Step 3: cross-operating

Step 4: assess and sort population pairs

Step 5: choose the best stocks of the NUM as a new parent population form Father and Son

Step 6: carry out mutation operation

Step 7: end if meet the training requirements and save the best results, otherwise, jump to Step 3

\section{Remarks and Related Works}

Heuristic Intelligent Planning we proposed in this paper is based on application-level knowledge .It can be extended to solve the general business problems. Since a Goal has many solutions, it is very important to choose the best one. Heuristic evaluation algorithm helps the user to choose an optimal implementation. Heuristic evaluation algorithm based on Genetic algorithm is a machine learning algorithm. It capture the experience gained during process planning and execution so as to enrich pattern library. And it can reduce the costs of business knowledge.

Pegasus [2,10] uses heuristic to search for an effective operator of the semiordered set in order to convert the current state to the target state. However, its planner and knowledge used for planning are tightly bound to a specific application domain. In our work, explicit knowledge representation (process patterns) is deployed to make workflow planner domain-independent.

The optimization method for process pattern proposed by Zhang Shaohua is based on the artificial neural network. As mentioned above, slow convergence and local minimum is a problem. We use the artificial neural network algorithm optimized by genetic algorithm to get out of this trouble.

\section{Conclusion}

Compared with process-driven workflow, goal-driven workflow generation based not on proprietary process definition frameworks, but on accepted ideas of how people actually work. And because the AI planning techniques had been integrated with workflow construction, goal-driven workflow generation enhanced the flexibility and adaptability of workflow system.

In this paper, a new workflow modeling method based on the process pattern is proposed to specify a goal-driven workflow correctly. This approach simplifies the logic and verification of the standard model, since process pattern is easier to understand and less complex to build. To avoid the users' intervention, a heuristic intelligent planning algorithm based on AI planning and genetic algorithm is deployed in this paper to generate workflow automatically. It provided a lightweight, efficient and cost-effective way to introduce knowledge into workflow composition. 
Our future work will concentrate on the following topics. The first one is to investigate algorithms to refine the undetermined elements during execution. The second one is to investigate re-planning algorithm and trace back method to handle the exception. The last one is to perfect machine learning algorithm to capture the experience gained during process planning and execution so as to enrich domain knowledge.

\section{References}

1. Alexander, C., Ishikawa, S., Silverstein, M., Jacobson, M., Fiksdahl King, I., Angel, S.: A Pattern Language. Oxford University Press, New York (1977)

2. Deelman, E., Singh, G., et al.: Pegasus: a Framework for Mapping Complex Scientific Workflows onto Distributed Systems. Scientific Programming Journal 13, 219-237 (2005)

3. Joeris, G.: Defining flexible workflow execution behaviors. In: Dadam, P., Reichert, M. (eds.) Enterprise-wide and Cross-enterprise Workflow Management: Concepts, Systems, Applications, CEUR Workshop Proceedings Paderborn, Germany, vol. 24 (1999)

4. http://software.idoxgroup.com/modules/idox_workflow.cfm

5. Basheer, I.A., Haimeer, M.: Artificial neural networks: fundamentals, computing, design, and application. Journal of Microbiological Methods 43, 3-31 (2000)

6. Jinlei, J., Shaohua, Z., Johann, S., Guangwen, Y.: Workflow Management in Grid Era: From Process-Driven Paradigm to a Goal-Driven One. In: Meersman, R., Tari, Z., Herrero, P. (eds.) OTM-WS 2007, Part I. LNCS, vol. 4805, pp. 169-178. Springer, Heidelberg (2007)

7. Kutluhan, E., James, H., Nau, D.S.: UMCP: A Sound and Complete Procedure for Hierarchical Task-Network Planning. AIPS, 249-254 (1994)

8. Rinderle, S., Reichert, M., Dadam, P.: Correctness criteria for dynamic changes in workflow systems: A survey. Data and Knowledge Engineering 50(1), 9-34 (2004)

9. van der Aalst, W.M.P., Weske, M., Grunbauer, D.: Case handling: A new paradigm for business process support. Data \& Knowledge Engineering 53(2), 129-162 (2005)

10. Kee, Y.S., Byun, E., Deelman, E., Vahi, K., Kim, J.S.: Pegasus on the Virtual Grid: A Case Study of Workflow Planning over Captive Resources. Workflows in Support of Large-Scale Science, 1-9 (2008)

11. Yong, X., Shaohua, Z., Yuzhu, S., et al.: Pattern-Oriented Workflow Generation and Optimization. Journal of Universal Computer Science 15(9), 1924-1944 (2009)

12. Shaohua, Z., Jinlei, J., Meilin, S., et al.: A Pattern-Oriented Planning Approach for Grid Workflow Generation. In: Proc. of Fifth International Conference on Grid and Cooperative Computing Workshops, pp. 275-281 (2006)

13. Shaohua, Z., Yong, X., Yuzhu, S., Meilin, S.: Knowledge Modeling and Optimization in Pattern-Oriented Workflow Generation. In: Proc. of the 12th International Conference on CSCW in Design, pp. 636-642 (2008) 